\title{
The Dark-Matter Density Profiles and Binary Black Holes
}

\author{
Li-Chin Yeh ${ }^{* i}$ \\ Department of Applied Mathematics, \\ National Hsinchu University of Education, Hsin-Chu City, Taiwan \\ E-mail: lcyeh@mail.nhcue.edu.tw

\section{Ing-Guey Jiang} \\ Department of Physics \& Institute of Astronomy, \\ National Tsing-Hua University, Hsin-Chu City, Taiwan \\ E-mail: jiang@phys.nthu.edu.tw
}

\begin{abstract}
It is known that galactic centers could host single or binary black holes, and observations show that black-hole masses are correlated with the properties of galaxies. Moreover, in terms of center's density profiles, early-type galaxies can be classified into power-law or core galaxies. Therefore, in this project, we study the dynamics near galactic centers with given galactic dark-matter density profiles and binary black holes. Our results could constrain the properties of binary black holes or dark-matter density profiles in their host galaxies.
\end{abstract}

Identification of Dark Matter 2010

July 26 - 302010

University of Montpellier 2, Montpellier, France

\footnotetext{
* Speaker.

${ }^{\dagger}$ We are grateful to the National Center for High-performance Computing for computer time and facilities. This work is supported in part by the National Science Council, Taiwan, under NSC 98-2112-M-007-006-MY2.
} 


\section{Introduction}

Black holes are always attractive for astronomers, and even for any scientists. Due to the improvement of observational techniques, it has been confirmed that black holes with different properties can exist in various cosmic environment. In addition to stellar mass black holes, one of the most popular classes is the super massive black holes in galactic centers. As many galaxies could have black holes in their centers, and galactic merging events take place very often during the formation and evolution of galaxies, it is likely that many galaxies would host more than one super massive black holes. These two super massive black holes could form a binary system in galactic centers and affect the nuclear density profile of galaxies. Assuming that the galactic centers are dominated by dark matter, here we study the dynamics near a black-hole binary in galactic centers with given density profiles of galaxies. We present our models in $\$ 2$ and the results in $\S 3$. The conclusions are in $\$ 4$.

\section{The Model}

We consider the motion of a test particle influenced by the gravitational force from the central binary black holes and the galaxy which is dominated by dark matter. We assume that two masses of the central binary black holes are $m_{1}$ and $m_{2}$ and choose the unit of mass to make $G\left(m_{1}+m_{2}\right)=1$, where $G$ is the gravitational constant. If we define that

$$
\mu=\frac{m_{2}}{m_{1}+m_{2}}
$$

then the two masses can be expressed as $\mu_{1}=G m_{1}=1-\mu$ and $\mu_{2}=G m_{2}=\mu$. The separation of central binary objects is set to be unity.

Under the rotating frame, the equation of motion for the test particle on $x-y$ plane is (Jiang \& Yeh 2006a)

$$
\left\{\begin{array}{l}
\frac{d x}{d t}=u \\
\frac{d y}{d t}=v \\
\frac{d u}{d t}=2 n v-\frac{\partial U^{*}}{\partial x}-\frac{\partial V}{\partial x} \\
\frac{d v}{d t}=-2 n u-\frac{\partial U^{*}}{\partial y}-\frac{\partial V}{\partial y},
\end{array}\right.
$$

where $n$ is the central binary's angular velocity,

$$
\begin{gathered}
U^{*}=-\frac{n^{2}}{2}\left(x^{2}+y^{2}\right)-\frac{1-\mu}{r_{1}}-\frac{\mu}{r_{2}} \\
r_{1}=\sqrt{(x+\mu)^{2}+y^{2}} \\
r_{2}=\sqrt{(x+\mu-1)^{2}+y^{2}},
\end{gathered}
$$

and $V$ is the potential from the galaxy. Since the black hole with mass $m_{1}$ is located at $(-\mu, 0)$, its distance to the test particle is $r_{1}=\sqrt{(x+\mu)^{2}+y^{2}}$. Similarly, the black hole with mass $m_{2}$ is at $(1-\mu, 0)$, its distance to the test particle is $r_{2}=\sqrt{(x+\mu-1)^{2}+y^{2}}$. We assume the density 
distribution satisfies the Nuker law (Lauer et al. 1995)

$$
\rho=\rho_{c}\left(\frac{r}{r_{b}}\right)^{-\gamma}\left\{1+\left(\frac{r}{r_{b}}\right)^{\alpha}\right\}^{\frac{\gamma-\beta}{\alpha}} .
$$

Here, we consider $\alpha=2, \beta=5$ and $\gamma=1$, the associated potential $V(r)$ satisfies

$$
V(r)=-\frac{c \rho_{c} r_{b}^{2}}{2}\left[\frac{\pi}{2}-\tan ^{-1}\left(\frac{r}{r_{b}}\right)\right]=-\frac{\bar{\alpha} r_{b}^{2}}{2}\left[\frac{\pi}{2}-\tan ^{-1}\left(\frac{r}{r_{b}}\right)\right],
$$

where $\bar{\alpha}=c \rho_{c}$, i.e. $c, \rho_{c}$ and $r_{b}$ are positive constants, and $r^{2}=x^{2}+y^{2}$.

\section{The Results}

\subsection{The Equilibrium Points when Central Binary's Angular Velocity $\mathbf{n}=\mathbf{1}$}

From Jiang \& Yeh (2006a, 2006b), equilibrium points should be either on the $x$-axis or on the line: $x=\frac{1}{2}-\mu$ of $x-y$ plane. As a simplification, we set angular velocity $n=1$ in this subsection. For equilibrium points on the $x$-axis, i.e. $\left(x_{e}, 0\right)$ and $r=\sqrt{x_{e}^{2}+0^{2}}=\left|x_{e}\right|$, we define

$$
\begin{aligned}
0 & =k_{1}\left(x_{e}\right)=x_{e}-\frac{(1-\mu)\left(x_{e}+\mu\right)}{\left|x_{e}+\mu\right|^{3}}-\frac{\mu\left(x_{e}+\mu-1\right)}{\left|x_{e}+\mu-1\right|^{3}}-\frac{x_{e}}{r} \frac{\partial V}{\partial r} \\
& =x_{e}-\frac{(1-\mu)\left(x_{e}+\mu\right)}{\left|x_{e}+\mu\right|^{3}}-\frac{\mu\left(x_{e}+\mu-1\right)}{\left|x_{e}+\mu-1\right|^{3}}-\frac{x_{e}}{\left|x_{e}\right|} \frac{\bar{\alpha} r_{b}^{3}}{2\left(r_{b}^{2}+x_{e}^{2}\right)} .
\end{aligned}
$$

On the other hand, for those equilibrium points on the line: $x=\frac{1}{2}-\mu$, i.e. $\left(\frac{1}{2}-\mu, y_{e}\right)$, and $r=\sqrt{\left(\frac{1}{2}-\mu\right)^{2}+y_{e}^{2}}$, we have

$$
\begin{aligned}
0 & =h_{1}\left(y_{e}\right)=1-\left(\frac{1}{4}+y_{e}^{2}\right)^{-\frac{3}{2}}-\frac{1}{r} \frac{\partial V}{\partial r} \\
& =1-\left(\frac{1}{4}+y_{e}^{2}\right)^{-\frac{3}{2}}-\frac{\bar{\alpha} r_{b}^{3}}{2\left\{r_{b}^{2}\left[\left(\frac{1}{2}-\mu\right)^{2}+y_{e}^{2}\right]^{\frac{1}{2}}+\left[\left(\frac{1}{2}-\mu\right)^{2}+y_{e}^{2}\right]^{\frac{3}{2}}\right\}}
\end{aligned}
$$

\section{Property 1}

There is one and only one $\overline{y_{1}}>0$ such that $h_{1}\left(\overline{y_{1}}\right)=0$, and only one $\overline{y_{2}}<0$ such that $h_{1}\left(\overline{y_{2}}\right)=0$. That is, there are two equilibrium points on the line: $x=\frac{1}{2}-\mu$.

We know that there are five equilibrium points for the classical restricted three-body problem. When there are more than five equilibrium points, we claim that new equilibrium points exist in this dynamical system. In Property 1, it is confirmed that there are two equilibrium points on the line: $x=\frac{1}{2}-\mu$. Through the following Property 2, we find that new equilibrium points could exist under some sufficient condition.

\section{Property 2}

(A) There is a point $x_{1}>1-\mu$ such that $k_{1}\left(x_{1}\right)=0$. 
(B) There is a point $0<x_{2}<1-\mu$ such that $k_{1}\left(x_{2}\right)=0$.

(C) If $\frac{\mu^{3}-(1-\mu)^{3}}{\mu^{2}(1-\mu)^{2}}+\frac{\bar{\alpha} r_{b}}{2}>0$, then there is a point $-\mu<x_{3}<0$ such that $k_{1}\left(x_{3}\right)=0$.

If $\frac{\mu^{3}-(1-\mu)^{3}}{\mu^{2}(1-\mu)^{2}}+\frac{\bar{\alpha} r_{b}}{2}<0$, then there is no $-\mu<x<0$ such that $k_{1}(x)=0$.

(D) There is a point $x_{4}<-\mu$ such that $k_{1}\left(x_{4}\right)=0$.

From the above Property 2, we know that when $\frac{\mu^{3}-(1-\mu)^{3}}{\mu^{2}(1-\mu)^{2}}+\frac{\bar{\alpha} r_{b}}{2}>0$, there are more equilibrium points near the center of this system. Thus, as long as the value of $\bar{\alpha}$ or $r_{b}$ is larger, i.e. a more massive host galaxy or a bigger central core of density profiles, there are six equilibrium points. In that case, this system might stay as a core galaxy and not evolve into a power-law one due to the existence of more equilibrium points near the galactic center.

\subsection{The Equilibrium Points when Central Binary's Angular Velocity $n=\sqrt{1-2 f_{b}(0.5)}$ and} $\mu=0.5$

In this subsection, we consider the case $\mu_{1}=\mu_{2}=0.5$. Under the gravitational influence from the galaxy, when $\mu_{1}=\mu_{2}=0.5$, both components of the central binary move on circular orbits at $r=0.5$ with mean motion, i.e. angular velocity $n=\sqrt{1-2 f_{b}(0.5)}$, (see Jiang \& Yeh 2006a, $2006 \mathrm{~b}$ ), where the gravitational force $f_{b}$ from the galaxy is

$$
f_{b}(r)=-\frac{\partial V}{\partial r}=\frac{-\bar{\alpha} r_{b}^{3}}{2\left(r_{b}^{2}+r^{2}\right)}
$$

and

$$
f_{b}(0.5)=\frac{-2 \bar{\alpha} r_{b}^{3}}{4 r_{b}^{2}+1}
$$

Hence,

$$
n=\sqrt{1-2 f_{b}(0.5)}=\sqrt{1+\frac{4 \bar{\alpha} r_{b}^{3}}{4 r_{b}^{2}+1}} .
$$

For convenience, we define

$$
\begin{aligned}
k^{\star}(x) & =n^{2} x-\frac{(x+0.5)}{2|x+0.5|^{3}}-\frac{(x-0.5)}{2|x-0.5|^{3}}+\left.\frac{x}{r} f_{b}(r)\right|_{(x, 0)} \\
& =\left(1+\frac{4 \bar{\alpha} r_{b}^{3}}{4 r_{b}^{2}+1}\right) x-\frac{(x+0.5)}{2|x+0.5|^{3}}-\frac{(x-0.5)}{2|x-0.5|^{3}}+\frac{x}{|x|}\left[\frac{-\bar{\alpha} r_{b}^{3}}{2\left(r_{b}^{2}+x^{2}\right)}\right] \\
h^{\star}(y) & =n^{2}-\left(\frac{1}{4}+y^{2}\right)^{-\frac{3}{2}}+\left.\frac{f_{b}(r)}{r}\right|_{(0, y)} \\
& =\left(1+\frac{4 \bar{\alpha} r_{b}^{3}}{4 r_{b}^{2}+1}\right)-\left(\frac{1}{4}+y^{2}\right)^{-\frac{3}{2}}-\frac{\bar{\alpha} r_{b}^{3}}{2|y|\left(r_{b}^{2}+y^{2}\right)} .
\end{aligned}
$$

In the following properties, we find that there are two equilibrium points on the $y$-axis and there are four equilibrium points on the $x$-axis.

\section{Property 3}

There is one and only one $\bar{y}_{3}>0$ such that $h^{\star}\left(\bar{y}_{3}\right)=0$, and only one $\bar{y}_{4}<0$ such that $h^{\star}\left(\bar{y}_{4}\right)=0$. 
That is, there are two equilibrium points on the line: $x=0$.

\section{Property 4}

(A) There is an $\bar{x}_{4}>0.5$ such that $k^{\star}\left(\bar{x}_{4}\right)=0$.

(B) There is an $0<\bar{x}_{5}<0.5$ such that $k^{\star}\left(\bar{x}_{5}\right)=0$.

(C) There is no $x=0$ such that $k^{\star}(x)=0$.

(D) There is an $-0.5<\bar{x}_{6}<0$ such that $k^{\star}\left(\bar{x}_{6}\right)=0$.

(E) There is an $\bar{x}_{7}<-0.5$ such that $k^{\star}\left(\bar{x}_{7}\right)=0$.

\section{Conclusions}

Depending on parameters of given galactic density profiles, number of equilibrium points could be different. According to the above four properties, which proof will be provided in our future papers, we have the results for the angular velocity $n=1$ case that, if sufficient condition in Property 2 is satisfied, i.e. $\frac{\mu^{3}-(1-\mu)^{3}}{\mu^{2}(1-\mu)^{2}}+\frac{\bar{\alpha} r_{b}}{2}>0$, there are six equilibrium points (four collinear and two triangular); if not, there are five equilibrium points (three collinear and two triangular). Moreover, for the second case, from Property 3 and Property 4, we find that there are always six equilibrium points.

In general, a system with a more massive host galaxy or a bigger central core of density profiles could have more equilibrium points. This might be related with whether this system will be a core galaxy or a power-law one. These dynamical results might be useful when one explores the density profiles of galactic centers and the properties of binary black holes.

\section{References}

[1] Lauer, T. R. et al., 1995, Astronomical Journal, 110, 2622

[2] Jiang, I.-G., Yeh, L.-C., 2006a, Astrophysics and Space Science, 305, 341

[3] Jiang, I.-G., Yeh, L.-C., 2006b, Astrophysics and Space Science, 306, 189 